\title{
Anhydrous Zinc Chloride: An efficient catalyst for one pot synthesis of 2,3,4,12-tetrahydro-benzo-[4,5]-thiazolo-[2,3b]-quinazolin-1-ones
}

\author{
Satish A. Dake, Sunil U.Tekale, Swapnil R. Sarda, Wamanrao N. Jadhav, Sudhakar R. Bhusare \\ and Rajendra P. Pawar* \\ Organic Chemistry Synthesis Laboratory, Dnyanopasak College, Parbhani-431401, India \\ E-mail:rppawar@yahoo.com
}

\begin{abstract}
One pot synthesis of quinazolin-1-one derivatives has been achieved from 2aminobenzothaizoles, 1,3-cyclohexanedione and aromatic aldehydes in presence of anhydrous zinc chloride catalyst. This methodology provides several advantages simple \& mild reaction conditions and excellent yield.
\end{abstract}

Keywords: Anhydrous zinc chloride, aldehydes, 2-aminobenzothaizoles, 1,3-cyclohexanedione, quinazolin-1-one

\section{Introduction}

In recent year heterocyclic compounds analogues of benzothaizoles and derivatives have attracted strong interest due to their useful biological and pharmacological properties. ${ }^{1-5}$ The chemistry of quinazolinones is interested because of its biological significance. Many of them shows antifungal, ${ }^{6}$ antibacterial, ${ }^{7}$ anticancer, ${ }^{8}$ anti-inflammatory, ${ }^{9}$ anticonvulsant ${ }^{10}$ and antiproliferative activities as well as inhibitory effects for thymidylate synthase and poly-(ADPribose) polymerase $(P A R P) .{ }^{11}$ Quinazolin-1-one derivatives are also found to be tranquilizer, antiallergic agent, an antiulcer agent and antiasthmatic agent. ${ }^{12}$

The common methods used for the preparation of such compounds are using

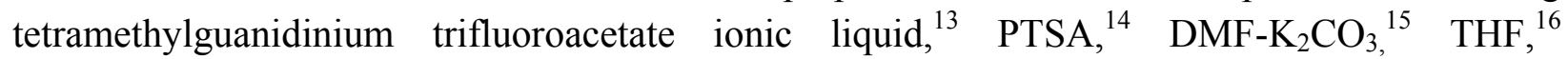
microwave irradiation. ${ }^{17}$ These methods have several limitations such as, pro-long reaction time, strong acidic condition and low yield. Consequently there is scope for further work on this reaction towards mild reaction condition, simple work up, and better yield. This has been achieved by using anhydrous zinc chloride as a catalyst to afford the corresponding quinazolin-1one in excellent yields. Herein we wish to report an efficient and mild protocol for the synthesis 2,3,4,12-tetrahydro-benzo-[4,5]-thiazolo-[2,3b]-quinazolin-1-one using zinc chloride as catalyst.

Recently, zinc chloride has received considerable attention as an inexpensive, readily available catalyst for various organic transformations. The Lewis acidity associated with zinc 
chloride enhanced rate of organic reaction and give excellent yield of the products with high selectivity. Owing to numerous advantages associated with this eco-friendly element, zinc chloride has been explored as a powerful catalyst in various organic transformations. ${ }^{18}$ Zinc chloride finds wide application in textile processing, metallurgical fluxes, and chemical synthesis. Anhydrous zinc chloride is used for number of reactions ${ }^{19}$ such as dehydration reaction, Beckmann rearrangement, synthesis of flavones, thiazoles, quinolines, nitrilies etc. Most of the reactions were completed within 4-9 hours affording 68-90\% yield of the product. In contrast, these reported methods required much longer reaction time and harsh reaction conditions. The $\mathrm{ZnCl}_{2}$-DMF system is found to be more suitable because of shorter reaction time and easy workup. But using other organic solvent systems it required longer reaction times. In absence of catalyst, the reaction does not proceed after longer time (15-20 h). The best result was obtained using 5-mol \% of zinc chloride in solvent dimethyl formamide at $120{ }^{\circ} \mathrm{C}$. Greater amount of the catalyst did not improve the yield. Solubility of zinc chloride catalyst in water provided an easy method for separation from the product.

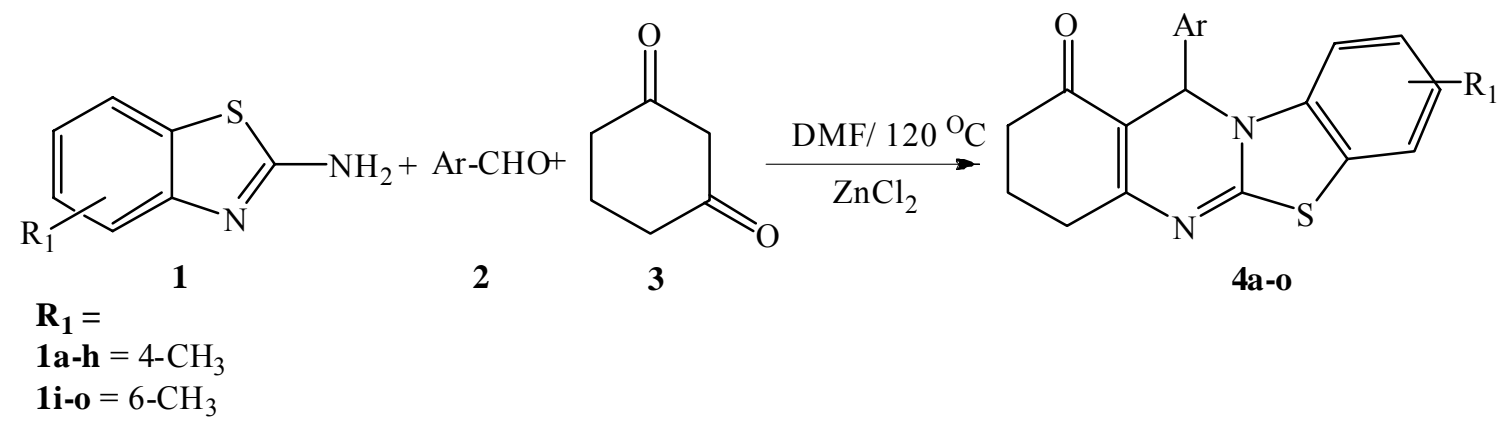

\section{Scheme 1}

\section{Results and Discussion}

In a model reaction 2-aminobezothiazoles (1), aromatic aldehyde (2), 1,3-cyclohexanedione (3) and zinc chloride catalyst in N,N-dimethyl formamide (DMF) was refluxed at $120{ }^{\circ} \mathrm{C}$ for a appropriate time. After completion of reaction, the usual work up offered the pure quinazolin-1one (4).

In conclusion, we demonstrated an efficient protocol for the synthesis of quinazolin-1-one derivatives using zinc chloride catalyst. Shorter reaction time, simple \& mild reaction conditions and higher yields render by this method. 
Table 1. Synthesis of benzo-[4,5]-thiazolo-[2,3-b]-quinazolin-1-one (4a-o)

\begin{tabular}{|c|c|c|c|c|c|}
\hline Entry & $\begin{array}{c}\mathrm{Ar}-\mathrm{CHO} \\
(2)\end{array}$ & $\begin{array}{c}\text { Products } \\
(4)\end{array}$ & $\begin{array}{l}\text { Reaction } \\
\text { Time (hr) }\end{array}$ & $\begin{array}{l}\text { M.P. } \\
\left({ }^{\circ} \mathrm{C}\right)\end{array}$ & $\begin{array}{l}\text { Yield } \\
(\%)^{\mathrm{a}}\end{array}$ \\
\hline $\mathbf{a}$ & & & 9.30 & 295 & 89 \\
\hline b & & & 11.30 & 98 & 85 \\
\hline C & & & 4.0 & 155 & 91 \\
\hline d & & & 4.30 & 112 & 79 \\
\hline e & & & 5.0 & 145 & 83 \\
\hline $\mathbf{f}$ & & & 7.0 & 80 & 86 \\
\hline g & & & 9.0 & 170 & 88 \\
\hline
\end{tabular}


Table 1. Continued

\begin{tabular}{|c|c|c|c|c|c|}
\hline Entry & $\begin{array}{c}\mathrm{Ar}-\mathrm{CHO} \\
(2)\end{array}$ & $\begin{array}{c}\text { Products } \\
(4)\end{array}$ & $\begin{array}{l}\text { Reaction } \\
\text { Time (hr) }\end{array}$ & $\begin{array}{l}\text { M.P. } \\
\left({ }^{\circ} \mathrm{C}\right)\end{array}$ & $\begin{array}{l}\text { Yield } \\
(\%)^{\mathrm{a}}\end{array}$ \\
\hline h & & & 9.0 & 150 & 92 \\
\hline i & & & 4.0 & 192 & 78 \\
\hline $\mathbf{j}$ & & & 8.0 & 130 & 80 \\
\hline $\mathbf{k}$ & & & 5.0 & 181 & 85 \\
\hline 1 & & & 7.0 & 125 & 89 \\
\hline $\mathbf{m}$ & & & 4.30 & 135 & 90 \\
\hline $\mathbf{n}$ & & & 9.0 & 150 & 91 \\
\hline
\end{tabular}


Table 1. Continued

\begin{tabular}{cccccc}
\hline Entry & $\begin{array}{c}\text { Ar-CHO } \\
(2)\end{array}$ & $\begin{array}{c}\text { Products } \\
(4)\end{array}$ & $\begin{array}{c}\text { Reaction } \\
\text { Time }(\mathrm{hr})\end{array}$ & $\begin{array}{c}\text { M.P. } \\
\left({ }^{\circ} \mathrm{C}\right)\end{array}$ & $\begin{array}{c}\text { Yield } \\
(\%)^{\mathrm{a}}\end{array}$ \\
\hline
\end{tabular}

${ }^{\mathrm{a}}$ Isolated yield.

\section{Experimental Section}

General procedure for the preparation of benzo-[4,5]-thiazolo-[2,3-b]-quinazolin-1-one (4a-o) To a mixture of 2-aminobenzothiazole (2.66 mmol), substituted benzaldehyde ( $2.66 \mathrm{mmol}), 1,3-$ cyclohexanedione $(2.66 \mathrm{mmol})$, and anhydrous zinc chloride $(5 \mathrm{~mol} \%)$ in $\mathrm{N}, \mathrm{N}$-dimethyl formamide $(5 \mathrm{~mL})$ solvent was added. The reaction mixture was refluxed at $120{ }^{\circ} \mathrm{C}$ in oil bath for appropriate time (Table 1). The progress of the reaction was monitored by TLC. After completion of reaction, the reaction mixture was poured onto ice-cold water with constant stirring. The separated solid product was filtered, washed with cold water and dried. The obtained crude was purified by column chromatography using dichloromethane and ethyl acetate in $3: 1$ ratio as an eluent.

10-methyl-12-(4-Hydroxy-3-methoxy-phenyl)-2,3,4,12-tetrahydro-benzo-[4,5]-thiazolo-[2,3b]quinazolin-1-one (4a). ${ }^{1} \mathrm{H} \mathrm{NMR}\left(\mathrm{CDCl}_{3}\right) \delta 1.18(2 \mathrm{H}$, quin), $2.29(2 \mathrm{H}, \mathrm{t}), 2.31(3 \mathrm{H}, \mathrm{s}), 2.74(2 \mathrm{H}$, t), $3.82(3 \mathrm{H}, \mathrm{s}), 10.18(1 \mathrm{H}, \mathrm{s}), 6.52(1 \mathrm{H}, \mathrm{s}), 7.30-7.60(6 \mathrm{H}, \mathrm{m}$, Ar-H). Anal. Calcd for $\mathbf{C}_{22} \mathbf{H}_{20} \mathbf{N}_{2} \mathbf{O}_{3} \mathrm{~S}$ (392.47): C, 67.33; H, 05.14; N, 07.14; O, 12.23; S, 08.17. Found: C, 67.30; H, 05.08; N, 07.18; O, 12.20; S, 08.19.

10-methyl-12-(4-Hydroxy-phenyl)-2,3,4,12-tetrahydro-benzo-[4,5]-thiazolo-[2,3b]-quinazolin1-one (4b). ${ }^{1} \mathrm{H}$ NMR $\left(\mathrm{CDCl}_{3}\right) \delta 1.16(2 \mathrm{H}$, quin), $2.32(2 \mathrm{H}, \mathrm{t}), 2.75(2 \mathrm{H}, \mathrm{t}), 2.38(3 \mathrm{H}, \mathrm{s}), 10.21$ $(1 \mathrm{H}, \mathrm{s}), 6.48(1 \mathrm{H}, \mathrm{t}), 7.23-7.62$ (7H, m, Ar-H). Anal. Calcd for $\mathbf{C}_{\mathbf{2 1}} \mathbf{H}_{\mathbf{1 8}} \mathbf{N}_{\mathbf{2}} \mathbf{O}_{\mathbf{2}} \mathbf{S}$ (362.44): C, 69.59; $\mathrm{H}, 5.01 ; \mathrm{N}, 7.73 ; \mathrm{O}, 8.83 ; \mathrm{S}, 8.85$. Found: C, 69.65; H, 04.98; N, 7.79; O, 8.81; S, 8.81.

10-methyl-12-(4-Chloro-phenyl)-2,3,4,12-tetrahydro-benzo-[4,5]-thiazolo-[2,3b]-quinazolin1-one (4c). ${ }^{1} \mathrm{H}$ NMR $\left(\mathrm{CDCl}_{3}\right) \delta 1.21(2 \mathrm{H}$, quin), $2.26(2 \mathrm{H}, \mathrm{t}), 2.33(3 \mathrm{H}, \mathrm{s}), 2.75(2 \mathrm{H}, \mathrm{t}), 6.37(1 \mathrm{H}$, s), 7.42-7.80 (7H, m, Ar-H). Anal. Calcd for $\mathbf{C}_{21} \mathbf{H}_{17} \mathbf{C l N}_{2} \mathbf{O S}$ (380.89): C, 66.22; H, 04.50; Cl, 09.31; N, 07.35; O, 04.20; S, 08.42. Found: C, 66.18; H, 04.48; Cl, 09.38; N, 07.32; O, 04.22; S, 08.40 .

10-methyl-12-(3-Hydroxy-phenyl)-2,3,4,12-tetrahydro-benzo-[4,5]-thiazolo-[2,3b]-quinazolin1-one (4d). ${ }^{1} \mathrm{H}$ NMR $\left(\mathrm{CDCl}_{3}\right) \delta 1.15(2 \mathrm{H}$, quin), $2.39(2 \mathrm{H}, \mathrm{t}), 2.42(3 \mathrm{H}, \mathrm{s}), 2.74(2 \mathrm{H}, \mathrm{t}), 9.88$ $(1 \mathrm{H}, \mathrm{s}), 6.62(1 \mathrm{H}, \mathrm{s}), 7.40-7.80(7 \mathrm{H}, \mathrm{m}, \mathrm{Ar}-\mathrm{H})$. 
10-methyl-12-(3-Nitro-phenyl)-2,3,4,12-tetrahydro-benzo-[4,5]-thiazolo-[2,3b]-quinazolin1-one (4e). ${ }^{1} \mathrm{H}$ NMR $\left(\mathrm{CDCl}_{3}\right) \delta 1.17(2 \mathrm{H}$, quin), $2.35(2 \mathrm{H}, \mathrm{t}), 2.41(3 \mathrm{H}, \mathrm{s}), 2.85(2 \mathrm{H}, \mathrm{t}), 6.53(1 \mathrm{H}$, s), 7.36-7.64 (7H, m, Ar-H). Anal. Calcd for $\mathbf{C}_{21} \mathbf{H}_{17} \mathbf{N}_{3} \mathbf{O}_{3} \mathbf{S}$ (391.44): C, 64.43; H, 04.38; N, 10.73; O, 12.26; S, 08.19. Found: C, 64.40; H, 04.35; N, 10.71; O, 12.24; S, 08.17.

10-methyl-12-(4-Styryl)-2,3,4,12-tetrahydro-benzo-[4,5]-thiazolo-[2,3b]-quinazolin-1-one (4f). ${ }^{1} \mathrm{H} \mathrm{NMR}\left(\mathrm{CDCl}_{3}\right) \delta 1.28(2 \mathrm{H}$, quin), $2.26(2 \mathrm{H}, \mathrm{t}), 2.32(3 \mathrm{H}, \mathrm{s}), 2.70(2 \mathrm{H}, \mathrm{t}), 6.43(1 \mathrm{H}, \mathrm{s})$, $6.80(1 \mathrm{H}, \mathrm{d}), 6.92(1 \mathrm{H}, \mathrm{d}), 7.42-7.76(8 \mathrm{H}, \mathrm{m}, \mathrm{Ar}-\mathrm{H})$.

10-methyl-12-(3-Bromo-4-hydroxy-5-methoxy-phenyl)-2,3,4,12-tetrahydro-benzo-[4,5]-thiazolo[2,3b]-quinazolin-1-one (4g). ${ }^{1} \mathrm{H} \mathrm{NMR}\left(\mathrm{CDCl}_{3}\right) \delta 1.17(2 \mathrm{H}$, quin), $2.25(2 \mathrm{H}, \mathrm{t}), 2.38(3 \mathrm{H}, \mathrm{s})$, $2.78(2 \mathrm{H}, \mathrm{t}), 3.88(3 \mathrm{H}, \mathrm{s}), 10.68(1 \mathrm{H}, \mathrm{s}), 6.66(1 \mathrm{H}, \mathrm{s}), 7.38-7.64(5 \mathrm{H}, \mathrm{m}, \mathrm{Ar}-\mathrm{H})$.

10-methyl-12-(2-Hydroxy-phenyl)-2,3,4,12-tetrahydro-benzo-[4,5]-thiazolo-[2,3b]-quinazolin1-one (4h). ${ }^{1} \mathrm{H}$ NMR $\left(\mathrm{CDCl}_{3}\right) \delta 1.20$ (2H, quin), $2.32(2 \mathrm{H}, \mathrm{t}), 2.36(3 \mathrm{H}, \mathrm{s}), 2.80(2 \mathrm{H}, \mathrm{t}), 9.48$ $(1 \mathrm{H}, \mathrm{s}), 6.60(1 \mathrm{H}, \mathrm{s}), 7.30-7.70(7 \mathrm{H}, \mathrm{m}, \mathrm{Ar}-\mathrm{H})$.

8-methyl-12-(4-Methoxy-phenyl)-2,3,4,12-tetrahydro-benzo-[4,5]-thiazolo-[2,3b]-quinazolin-1one (4i). ${ }^{1} \mathrm{H}$ NMR $\left(\mathrm{CDCl}_{3}\right) \delta 1.24(2 \mathrm{H}$, quin), $2.38(2 \mathrm{H}, \mathrm{t}), 2.42(3 \mathrm{H}, \mathrm{s}), 2.74(2 \mathrm{H}, \mathrm{t}), 3.86(3 \mathrm{H}$, s), $6.62(1 \mathrm{H}, \mathrm{s}), 7.38-7.72(7 \mathrm{H}, \mathrm{m}, \mathrm{Ar}-\mathrm{H})$.

8-methyl-12-(2-Hydroxy-phenyl)-2,3,4,12-tetrahydro-benzo-[4,5]-thiazolo-[2,3b]-quinazolin-1one (4j). ${ }^{1} \mathrm{H}$ NMR $\left(\mathrm{CDCl}_{3}\right) \delta 1.24(2 \mathrm{H}$, quin), $2.30(2 \mathrm{H}, \mathrm{t}), 2.48(3 \mathrm{H}, \mathrm{s}), 2.72(2 \mathrm{H}, \mathrm{t}), 9.80(1 \mathrm{H}$, s), $6.46(1 \mathrm{H}, \mathrm{s}), 7.36-7.72(7 \mathrm{H}, \mathrm{m}, \mathrm{Ar}-\mathrm{H})$.

8-methyl-12-(3-Methoxy-4-hydroxy-phenyl)-2,3,4,12-tetrahydro-benzo-[4,5]-thiazolo-[2,3b]quinazolin-1-one (4k). ${ }^{1} \mathrm{H} \mathrm{NMR}\left(\mathrm{CDCl}_{3}\right) \delta 1.20(2 \mathrm{H}$, quin), $2.30(2 \mathrm{H}, \mathrm{t}), 2.36(3 \mathrm{H}, \mathrm{s}), 2.78(2 \mathrm{H}$, t), $3.89(3 \mathrm{H}, \mathrm{s}), 10.36(1 \mathrm{H}, \mathrm{s}), 6.52(1 \mathrm{H}, \mathrm{s}), 7.46-7.80(6 \mathrm{H}, \mathrm{m}, \mathrm{Ar}-\mathrm{H})$.

8-methyl-12-(3-Nitro-phenyl)-2,3,4,12-tetrahydro-benzo-[4,5]-thiazolo-[2,3b]-quinazolin-1one (4l). ${ }^{1} \mathrm{H}$ NMR $\left(\mathrm{CDCl}_{3}\right) \delta 1.16(2 \mathrm{H}$, quin), $2.34(2 \mathrm{H}, \mathrm{t}), 2.44(3 \mathrm{H}, \mathrm{s}), 2.77(2 \mathrm{H}, \mathrm{t}), 6.60(1 \mathrm{H}$, s), 7.20-7.48, (7H, Ar-H).

8-methyl-12-(Furyl)-2,3,4,12-tetrahydro-benzo-[4,5]-thiazolo-[2,3b]-quinazolin-1-one (4m). ${ }^{1} \mathrm{H}$ NMR $\left(\mathrm{CDCl}_{3}\right) \delta 1.14(2 \mathrm{H}$, quin), $2.28(2 \mathrm{H}, \mathrm{t}), 2.30(3 \mathrm{H}, \mathrm{s}), 2.70(2 \mathrm{H}, \mathrm{t}), 6.62(1 \mathrm{H}, \mathrm{s}), 7.32-$ $7.52(3 \mathrm{H}, \mathrm{m}, \mathrm{Ar}-\mathrm{H}), 7.78-7.88(3 \mathrm{H}, \mathrm{m}, \mathrm{Ar}-\mathrm{H})$.

8-methyl-12-(2-Chloro-phenyl)-2,3,4,12-tetrahydro-benzo-[4,5]-thiazolo-[2,3b]-quinazolin1-one (4n). ${ }^{1} \mathrm{H}$ NMR $\left(\mathrm{CDCl}_{3}\right) \delta 1.18(2 \mathrm{H}$, quin), $2.28(2 \mathrm{H}, \mathrm{t}), 2.30(3 \mathrm{H}, \mathrm{s}), 2.72(2 \mathrm{H}, \mathrm{t}), 6.50$ $(1 \mathrm{H}, \mathrm{s}), 7.32-7.58(7 \mathrm{H}, \mathrm{m}, \mathrm{Ar}-\mathrm{H})$.

8-methyl-12-(2-Hydroxy-4-chloro-phenyl)-2,3,4,12-tetrahydro-benzo-[4,5]-thiazolo-[2,3b]quinazolin-1-one (4o). ${ }^{1} \mathrm{H} \mathrm{NMR}\left(\mathrm{CDCl}_{3}\right) \delta 1.18(2 \mathrm{H}$, quin), $2.28(2 \mathrm{H}, \mathrm{t}), 2.32(3 \mathrm{H}, \mathrm{s}), 2.74(2 \mathrm{H}$, t), $10.38(1 \mathrm{H}, \mathrm{s}), 6.50(1 \mathrm{H}, \mathrm{s}), 7.30-7.48(6 \mathrm{H}, \mathrm{m}, \mathrm{Ar}-\mathrm{H})$.

\section{Acknowledgements}

The authors are thankful to the Principal Dr. P. L. More Dnyanopasak College, Parbhani, for encouragement during the process of carrying out this work. 


\section{References}

1. Kappe, C. O. Tetrahedron 1993, 49, 6963.

2. (a) Mase, T.; Arima, H.; Tomioka, K.; Yamada, T.; K. Murase, J. Med. Chem. 1988, 29, 386. (b) G. Trapani; M. Franco; A. Latrofa; Genchi, G.; Liso, G. J. Med. Chem. 1992, 15, 39. (c) Wade, J. J.; Toso, C. B.; Matson, C. J.; Stelzer, V. L. J. Med. Chem. 1983, 26, 608.

3. Trapani, G.; Franco, M.; Latrofa, A.; Iacobazzi, V.; Ghiani, C. A.; Maciocco, E.; Liso, G. Eur. J. Med. Chem. 1997, 32, 83.

4. Ward, C. E.; Berthold, R. V.; Koerwer, J.; Tomlin, J. B.; Manning, D. T. J. Agric. Food Chem. 1986, 43, 1005.

5. Alajarin, R.; Jordan, P.; Vaquero, J. J.; Alvarez-Builla, J. Synthesis 1995, 389.

6. Sawhney, S. N; Tower, R. K; Singh, S. P. Indian J. Chem. 1980, 19, 415.

7. Ana, B; Boteanu, S. Farmacia 1971, 19, 683.

8. Dempcy, R. O; Skibo, E. B. Bioorg. Med. Chem. Lett. 1993, 1, 39.

9. Bhargava, P. N; Singh, G. C. J. Indian Chem. Soc. 1961, 38, 77.

10. Gujral, M. L; Saxena, P. N; Kohli, R. P. Indian J. Med. Res. 1957, 45, 201.

11. Griffin, J; Srinivasan, S; Bowman, K; Kalvert, H. A; Curtin, N. J; Newel, D. R; Pemberton, L. C; Golding, B. T. J. Med. Chem. 1998, 41, 5247.

12. (a) Awoutrs, F; Vermeire, J; Smeyers, F; Vermote, T; Van Beek, R; Niemgeers, C. J. E. Drugs Dev. Res. 1980, 8, 95. (b) Matsutani, S; Mizushima, Y. Chem. Abstr. 1990, 112, 985557. (c) Yanagihara, Y; Kasai, H; Kawashime, T; Shaida, T. Japan J. Pharmacol. 1988, 48, 91.

13. Shaabani, A; Rahmati, A., Naderi, S. Bioorg. Med. Chem. Lett. 2005, 15, 5553.

14. Trapani, G; Franco, M; Latrofa, A; Carotti, A; Genchi, G; M; Serra, Biggio, G; Liso, G; Eur. J. Med. Chem. 1996, 31, 575.

15. Pingle, M. S; Vartale, S. P; Bhosale, V. N; Kubekar, S. V. Arkivoc 2006, (x), 190.

16. Trapani, G; Franco, M; Latrofa, A; Genchi, G; Iacobazzi, V; Ghiani, C. A; Maciocco, E; Liso, G. Eur. J. Med. Chem. 1997, 32, 83.

17. Mourad, A. F. E; Aly, A. A; Farag, H. H; Beshr, E. A. Beilstein J. Org. Chem. 2007, 3, 11.

18. Ahmed, M. G; Ahmed, S. A; Romman, U. K. R; Sultana, T; Rahman, M. B; Hossain, M. A; Uddin, K. Indian J. Chem. 2005, 44, 622.

19. (a) Hu, E; Sidler, D. R; Dolling, U. J. Org. Chem. 1998, 63, 3454. (b) Varma, R. S; Saini, R. K. Synlett 1997, 857-858. (c) Varma, R. S; Dahiya, R; Kumar, S. Tetrahedron Lett. 1997, 38, 2039. (d) Varala, R; Enugala, R; Srinivas, R. Arkivoc 2006, (xiii), 171. (e) Nagy, N. M; Jakab, M. A; Kónya, J; Antus, S. Applied Clay Science 2002, 21, 213. (f) SampathKumar, H. M; Anjaneyulu, S; Subba Reddy, B. V; Yadav, J. S. Synlett 2000, 487. 